\title{
Crystallize Nanoparticles by Precipitating Trace Polymeric Additives
}

Yiwen Qian ${ }^{1,2}$, Alessandra Da Silva ${ }^{3}$, Emmy Yu $^{4}$, Christopher L. Anderson ${ }^{4,5}$, Yi Liu ${ }^{5}$, Wolfgang Theis ${ }^{3}$, Peter Ercius ${ }^{6}$, and Ting $\mathrm{Xu}^{1,2,4^{*}}$

${ }^{1}$ Department of Materials Science and Engineering, University of California, Berkeley, CA 94720, USA

${ }^{2}$ Materials Sciences Division, Lawrence Berkeley National Laboratory, Berkeley, CA 94720, USA

${ }^{3}$ Nanoscale Physics Research Laboratory, School of Physics and Astronomy, University of Birmingham, Birmingham B15 2TT, UK

${ }^{4}$ Department of Chemistry, University of California, Berkeley, CA 94720, USA

${ }^{5}$ The Molecular Foundry, Lawrence Berkeley National Lab, Berkeley, CA 94720, USA

${ }^{6}$ National Center for Electron Microscopy, Lawrence Berkeley National Laboratory, Berkeley, CA 94720, USA

*Corresponding author email: tingxu@berkeley.edu 


\begin{abstract}
Growing nanoparticle (NP) crystals has been pursued extensively using ligand chemistries such as DNA and supramolecules, controlled evaporation and patterned surfaces. Here, we show that a trace amount of polymeric impurities $(<0.1 \mathrm{wt} . \%)$ leads to reproducible, rapid growth of high quality 3-D NP crystals in solution and on patterned substrates with high yield. The polymers preferentially precipitate on the NP surfaces inducing the formation of small NP clusters, which subsequently act as nuclei to initiate NP crystal growth in dilute solution. This precipitationinduced NP crystallization process is applicable for a range of polymers and the resultant 3-D NP crystals can be tuned by varying polymeric additives loading, solvent evaporation rate and NP size. Fundamentally, the present study elucidates how to balance cohesive energy density and NP diffusivity in the self-assembly to favor nuclei formation energetically and kinetic growth in dilute solutions. The results shown also opened up the process window to rapidly and reliably fabricate NP crystals over multiple length scales. Furthermore, the amount of these impurities needed to grow NP crystals $(<0.1 \%)$ reminds us the need to pay special attention to fine details to interpret experimental observations in nanoscience.
\end{abstract}




\section{Introduction}

Crystallization is a ubiquitous process seen in most, if not all, classes of matter. Nanoparticles (NPs) are ideal to visualize and understand the crystallization processes ${ }^{1,2}$ and as building blocks toward new classes of materials ${ }^{3,4}$. A dazzling array of NP crystals have been demonstrated by engineering complimentary interactions, e.g. by attaching DNA ligands ${ }^{5-7}$. Maintaining adequate system mobility is requisite to achieve highly ordered NP assemblies. While the initial stages of the nucleation process remain debatable, it is commonly accepted that the critical nucleation size depends on the balance between the NP/solvent interfacial interactions and NP/NP cohesive energy stored within the nucleus ${ }^{8}$. Nuclei are energetically stable at elevated NP concentrations ${ }^{9}$ and/or with strong ligand interactions. The NP mobility scales inversely with the NP concentration and strong ligand pair interactions. Different strategies have been employed to open the process window to crystalize NPs, including controlled solvent evaporation, interface mediated assembly ${ }^{10-12}$, emulsion-based assembly ${ }^{13,14}$ and slowly cooling in concentrated/supersaturated solutions ${ }^{15}$. However, it remains challenging to control the crystallization kinetics so that high quality NP crystals can be rapidly and reliably fabricated.

Precipitating agents such as salts, solvents and polymers have been used to drive and accelerate the crystallization of small molecules and proteins ${ }^{16-19}$. These additives can alter the molecular interactions to either stabilize the intermediate phase ${ }^{19,20}$ or to reduce the solubility of crystallizing species $^{21}$. The application of a similar concept to NP crystallization may lead to control over the assembly kinetics and pathway, but has not yet been experimentally explored.

Here we hypothesize that by introducing polymers with poor solubility, NP surfaces may be the preferred precipitating sites to form small NP clusters, which will subsequently act as nuclei to initiate NP crystallization in a dilute solution (Fig. 1a). Given sufficient NP mobility, rapid NP 
crystallization can be realized while achieving a high degree of crystalline order. Here, we tested this hypothesis and experimentally realized polymer precipitant-induced crystallization of polymer-grafted nanoparticles (PGNPs). 3-D PGNP clusters can be obtained rapidly (in a few to tens of minutes) using a range of polymers, including impurities from plastic containers. The polymeric precipitants indeed led to small PGNP clusters with poor local order. These clusters subsequently grew into large 3-D PGNP crystals. Detailed tomography analysis of a 3-D PGNP crystal with single particle resolution confirmed the presence of defects and less positional order of individual NPs suggesting rather weak cohesive energy and local NP diffusion. This precipitating additive approach is facile where the PGNP crystal formation depends on polymeric additive loading, solvent evaporation rate, PGNP size and nucleation sites. As an example, a patterned substrate was used to grow hierarchically ordered arrays of PGNP crystals with controlled size, location and orientation.

\section{Results}

PGNPs were chosen as the building block due to their programmability and diversity in ligand chemistry $^{22-29}$. Gold NPs grafted with thiol end-functionalized polystyrene (PS) ligands were well dispersed in toluene. A series of polymers were employed as precipitates with non-favorable interactions with toluene and the PS ligand. Figure 1b shows the TEM images of PGNP crystals in the presence of hydrophilic homopolymers, hydrophobic homopolymers and block-copolymers, respectively. Control experiments without polymer precipitants as detailed in the Supplementary Information (SI) (Fig. S12-13) showed only 2-D interfacial PGNP assemblies, consistent with previous results ${ }^{24}$. Interestingly, we found that the polymers dissolved from a polyolefin centrifuge tube turned out to be the best precipitants to grow high-quality 3-D PGNP crystals reliably and 
rapidly. The polymer additive, henceforth called $\mathrm{PP} / \mathrm{PE}$, is chemically identified as a low molecular weight, non-crystalline polypropylene (PP) with $\sim 10 \%$ of polyethylene $(\mathrm{PE})$ as detailed in SI S2.

a

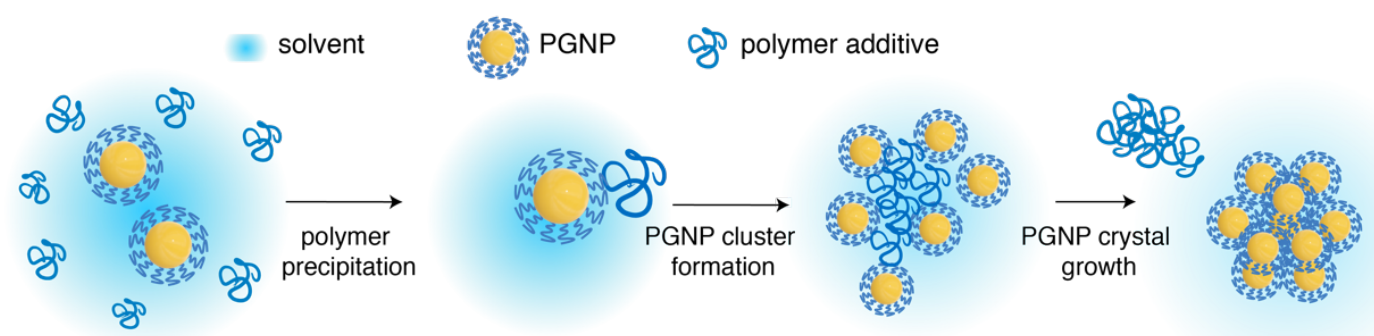

b

PNIPAM-COOH

$\mathrm{PBd}-\mathrm{COOH}$

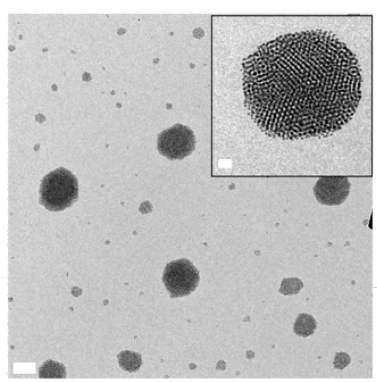

C
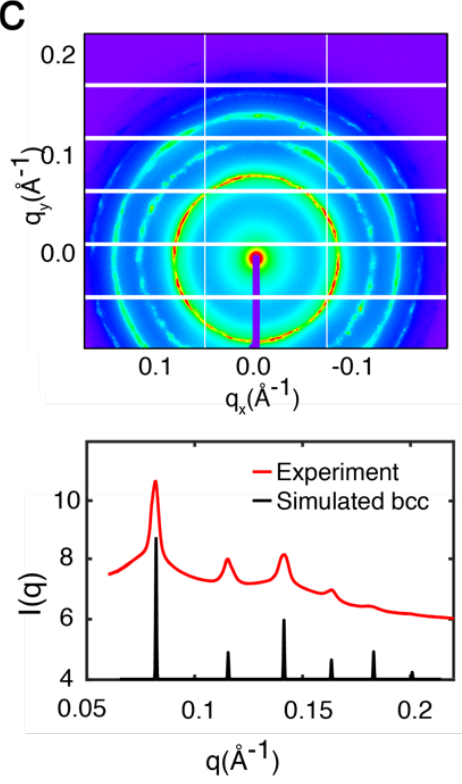

d

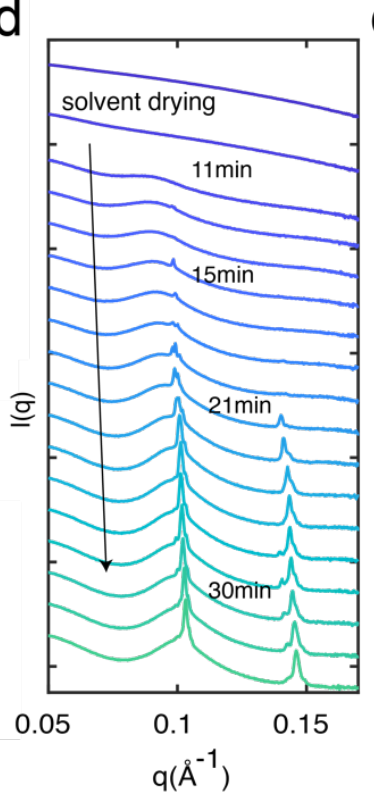

e

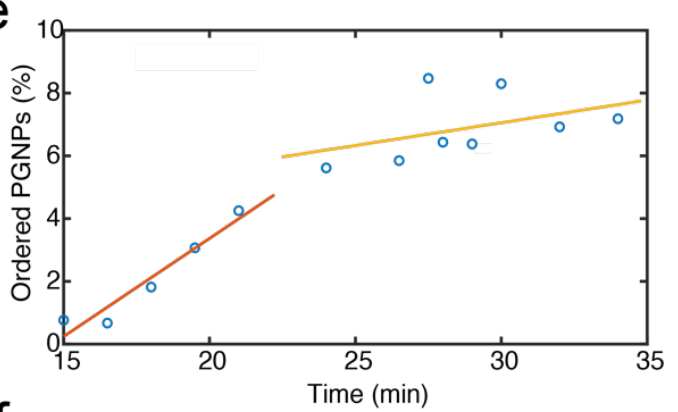

f

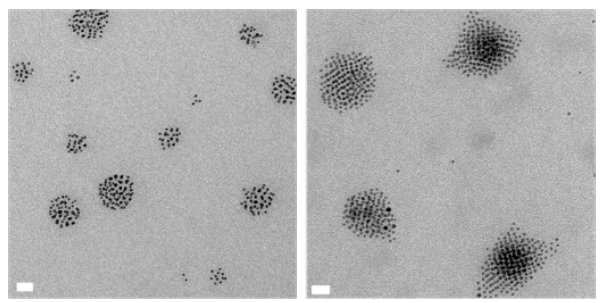

Fig. 1 | Polymer precipitation induced PGNP crystallization in solution during solvent evaporation. a, Schematic of polymer precipitant-induced PGNP crystallization: the added polymers precipitate onto PGNP surfaces to form small PGNP clusters that grow into crystals. b, 
Representative TEM images of PGNP assemblies of (1.7k) Au-PS 0.70 NPs with various polymer precipitants. See methods for detailed polymer information. Scale bars are $100 \mathrm{~nm}$ for PNIPAM$\mathrm{COOH}$, PS-b-PHEMAC, $1 \mu \mathrm{m}$ for PBd-COOH, PS-b-PHEMAC, PP/PE and 20nm for all inset images. c, SAXS results of a PGNP crystals in toluene solution of (5.3k) Au-PS 0.60 NPs with PP/PE. A simulated scattering profile of a perfect bcc lattice is shown for reference. d, In-situ SAXS studies of toluene solutions of (1.7k) Au-PS ${ }_{0.70}$ NPs and PP/PE during drying process. Plots are offset for clarity. e, PGNP crystal growth in toluene solution of (1.7k) Au-PS 0.70 NPs and PP/PE over time. Data points reflect the peak integration ratio between the sharp diffraction peak at $\mathrm{q} \sim$ $0.1 \AA^{-1}$ in $\mathbf{d}$ from PGNPs crystals and the broad peak from PGNPs in the solution. $\mathbf{f}$, TEM images of (1.7k) Au-PS ${ }_{0.70} \mathrm{NP}$ assemblies with PP/PE upon fast solvent removal. Scale bars: $20 \mathrm{~nm}$. PGNPs were dispersed at $\emptyset_{N P}=0.1 \mathrm{vol} \%$ with $\emptyset_{P P / \mathrm{PE}}=0.08 \mathrm{vol} \%$ and all other polymers at $\emptyset_{\text {polymer }}=0.2$ vol $\%$.

\section{Polyolefin Precipitation Leads to PGNP Crystallization in Solution}

$\mathrm{Au}$ PGNPs (diameter $=3.8 \pm 0.4 \mathrm{~nm}$ ) denoted as $(5.3 \mathrm{k})$ Au-PS $\mathrm{P}_{0.60}$ NPs (with PS numberaverage molecular weight $\mathrm{Mn}=5,300 \mathrm{~g} / \mathrm{mol}$, grafting density $\Sigma=0.60$ chains $/ \mathrm{nm}^{2}$ ), were dispersed in toluene at $\emptyset_{N P}=0.1 \mathrm{vol} \%$. PP/PE is added at $\emptyset_{P P / P E}=0.08 \mathrm{vol} \%$. As the toluene evaporated, small-angle X-ray scattering (SAXS) profiles showed multiple well-defined diffraction peaks with peak positions at $q_{n}: q_{1}=1: \sqrt{2}: \sqrt{3}: 2: \sqrt{5}: \sqrt{7}$, consistent with a bodycentered-cubic (bcc) structure (Fig. 1b). In-situ SAXS was carried out to monitor the PGNP assembly during solvent evaporation (Fig. 1c). The solution contains Au PGNPs denoted as (1.7k) Au-PS 0.70 NPs (with PS Mn=1,680 kg/mol and $\sum=0.70$ chains $/ \mathrm{nm}^{2}$ ) and PP/PE at volume fraction $\emptyset_{P P / P E}=0.08 \%$. At $\emptyset_{N P} \sim 23 \%(\mathrm{t}=11 \mathrm{~min})$, the PGNP diffusion coefficient was estimated to be $\sim 2.96 \times 10^{-11} \mathrm{~m}^{2} / \mathrm{s}$ using Stokes-Einstein equation: $D=\frac{k_{B} T}{6 \pi \eta r}(1)$, where $\eta$ is the dynamic viscosity and $\mathrm{r}$ is the radius of a spherical particle. The scattering profile showed a broad peak, corresponding to an average interparticle distance of $6.8 \mathrm{~nm}$. This peak originates from the poorly- 
ordered PGNP clusters since the average PGNP separation distance is $\sim 7.8 \mathrm{~nm}$ at this $\emptyset_{N P}$. At $\mathrm{t}$ $=15 \mathrm{~min}$, an additional sharp diffraction peak appeared at $q_{1}=0.10 \AA^{-1}$. The peak intensified with a $2^{\text {nd }}$ order peak appearing at $q_{1}: q_{2}=1: \sqrt{2}$, confirming the formation of well-ordered PGNP assemblies. The intensity of the diffraction peaks increased and shifted to higher q positions as the solvent evaporated. The final interparticle distance was $6.1 \mathrm{~nm}$ and the estimated domain size, derived from the peak-width, was $\sim 450 \mathrm{~nm}$. The broad and the sharp diffraction peaks were deconvoluted to probe the PGNP crystal growth as detailed in SI. As the solvent evaporated, an increasing fraction of PGNPs assembled into ordered structures. Based on the estimated PGNP diffusion coefficient, the PGNP crystal formation relies on short-range diffusion. The NP crystals grew rapidly first and then slowed down as shown in Fig. 1d. This can be attributed to the decrease of the PGNP diffusivity as the solvent evaporated and again highlights the importance of NP mobility in dilute solutions for crystal growth.

By using a small amount of solution $(<5 \mu \mathrm{L})$, the self-assembly was quenched at the early stage. Both NP assemblies with high crystalline order and poor order were seen using TEM. Image analysis confirmed that NP assemblies less than $30 \mathrm{~nm}$ in diameter consistently showed poor structural ordering (Fig. 1e) while larger NP clusters exhibited crystalline structures. Control experiments confirmed the absence of 3-D well-ordered NP crystals without the addition of polyolefin, and only 2-D superlattices were found (SI Fig. S12-13). Thus, the poorly ordered NP clusters may very likely still contain polyolefin precipitants but nevertheless can act as nuclei for subsequent NP crystallization. Recent work showed that an amorphous, liquid-like intermediate phase exists within the crystallization process, in agreement with our experimental observation ${ }^{2}$. Further SAXS and TEM analysis confirmed that the inter-NP distance remained the same between 
the PGNP assemblies with and without added polyolefins. Thus, it is reasonable to conclude that polyolefins were excluded during subsequent crystallization process.

\section{Effects of Polymer Precipitates and Solvent Evaporation on Crystal Growth}

The amount of polyolefin added affects the size of the PGNP crystals formed. Figure 2a shows representative TEM images of the crystals at different PP/PE loading $\left(\emptyset_{P P / P E}\right)$. At low $\emptyset_{P P / P E}(<$ 0.01\%), layered 2D superlattices with small grain sizes were seen. 3-D PGNP superlattices formed when $\emptyset_{P P / P E}$ reached $0.05 \%$. At $\emptyset_{P P / P E} \sim 0.5 \%$, PGNPs assembled into larger crystals with multiple grains. As more polyolefin was added, there was an increase in the average crystal diameter and size distribution (Fig. 2b and SI Fig. S19). The larger sizes of assemblies correspond to a smaller total surface area that reduces the non-favorable interactions between the PGNP surface and polyolefins. The fact that the larger-sized crystals formed with more polyolefins suggests that after the nuclei formation, polyolefins are excluded from the bulk crystal, interact with the crystal surface and control the sizes of the final crystals formed. This is consistent with the unchanged inter-particle distance with polymeric additives.

The polymeric ligands on PGNPs are critical for the nucleation and growth of PGNP crystals. In control experiments using oleylamine (OAm) stabilized Au NPs, no ordered 3-D crystals were observed (SI Fig. S14). The co-assembly of PGNPs and OAm-NPs resulted in crystallization of only the PGNPs, with the OAm-stabilized NPs excluded (SI Fig. S15). The spontaneous phase separation between NPs with two different ligand chemistries confirmed the importance of PS ligand contributions toward PGNP crystal growth. These results support our hypothesis that the polyolefin preferentially precipitates on NPs with polymeric ligands, and thus modulates the PGNP nucleation and crystallization. 
PGNP crystal growth depends on the solvent evaporation process. Grazing incidence/transmission small angle x-ray scattering (GI(T)SAXS) was performed to monitor the growth of PGNPs on the Si wafer upon solvent evaporation when $\emptyset_{P P / P E}$ was set to $0.15 \%$. As a transmission technique, GI(T)SAXS probes entire assemblies. The scattering patterns of the final dried samples are shown (Fig. 2c). At a solvent evaporation rate of $\sim 1 \mu \mathrm{L} / \mathrm{min}$, diffraction spots appeared at an incidence angle of $\alpha=0.8^{\circ}$. The peaks are assigned to be the diffraction patterns of a bcc crystal lattice with zone axes of [111], [100], [011], and [311] (SI Fig. S17). The presence of defined peaks indicates that the bcc crystals formed with (110) planes aligned parallel to the substrate throughout the whole sample (Fig. 2d). Thus, the crystals formed during solvent evaporation are strongly biased by the substrates due to the low cohesive energy of PGNP clusters $^{30}$. There was no diffraction spot when $\alpha=0.14^{\circ}$ (SI Fig. S18), meaning PGNP crystals were not formed directly at the $\mathrm{Si}$ substrate/PGNP interfaces ${ }^{31}$, again differentiating with the previously reported NP interfacial assembly mechanism ${ }^{10,11,24}$.

When the solvent evaporation rate was increased to $2.5 \mu \mathrm{L} / \mathrm{min}$, very few ordered assemblies formed, indicating that the PGNPs did not have enough mobility to organize into crystals. On the other hand, when the solvent evaporation rate was decreased to $0.7 \mu \mathrm{L} / \mathrm{min}$, a combination of aligned crystals, 2D layers, and randomly oriented crystals were formed, and the resulting scattering patterns were a combination of both diffraction spots and vertical lines. Layered superlattices are typically found when the PGNPs assemble at interfaces without polyolefins. They are formed due to the capillary force ${ }^{32}$ and preferential lateral diffusion. As the PGNP flux is low ${ }^{33}$ at low solvent evaporation rates, PGNPs close to the solvent drying front are able to stack at the droplet-substrate interfaces to form 2-D layers, while others continue to grow into 3-D PGNP crystals. As the PP/PE loading is increased, however, the precipitant overwhelms the substrate 
effect, and randomly oriented crystals formed. A phase diagram is plotted to map out the morphologies formed with varying amounts of polymeric additives and differing solvent evaporation rates (Fig. 2e).

With a controlled PP/PE amount and solvent evaporation rate, single-crystalline superlattice clusters with preferred orientation form exclusively upon solvent evaporation $(<20 \mathrm{~min})$ on

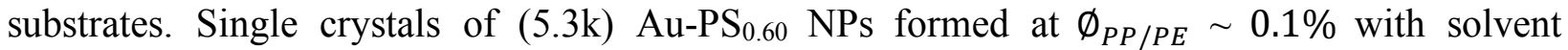
evaporation rate of $0.8 \mu \mathrm{L} / \mathrm{min}$. Multiple TEM images are provided to demonstrate the high-yield crystal formation. Each of the crystals has distinctive rod-like contour shape and clear bcc packing, supported by the diffraction spots in fast Fourier transform (FFT) (SI Fig. S20).

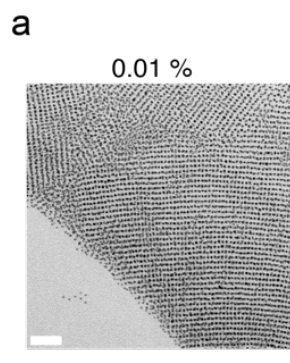

C

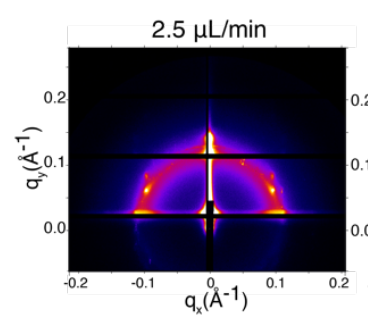

Polyolefin volume fraction $\left(\phi_{\text {PPPE }}\right)$

$0.05 \%$

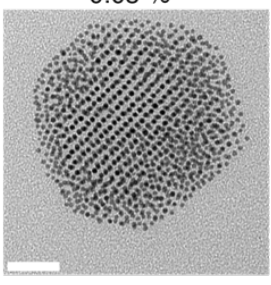

Solvent evaporation rate

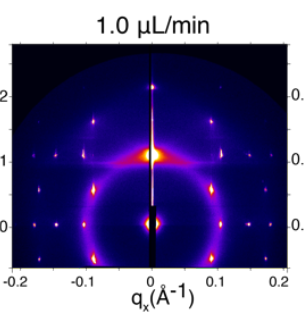

$0.1 \%$
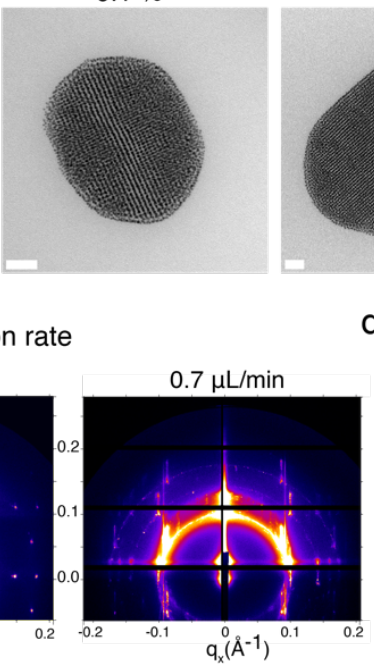

b

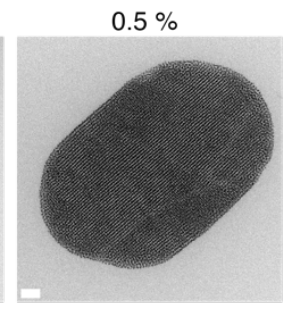

d

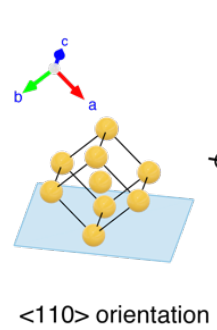

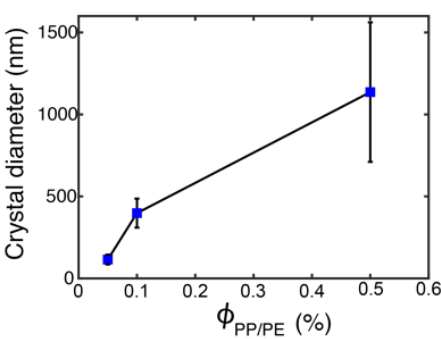

e

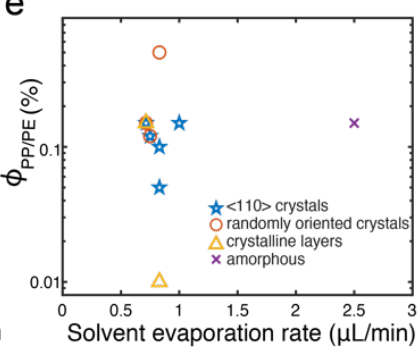

Fig. 2 Effects of polyolefin loading and solvent evaporation rate on crystal growth. a, TEM images of the self-assembled (5.3k) Au-PS ${ }_{0.60}$ NPs with different volume fraction of PP/PE. Scale bar is $50 \mathrm{~nm}$ for $\emptyset_{P P / P E} \sim 0.01 \%, 0.05 \%$ and $0.1 \%$ and $200 \mathrm{~nm}$ for $0.5 \%$. b, Crystal size as a function of polyolefin loading. Crystals analyzed were assembled using (5.3k) Au-PS 0.60 NPs with different volume fraction of PP/PE. c, Two-dimensional GI(T)SAXS patterns of the assembled (1.7k) Au-PS ${ }_{0.70}$ NPs with PP/PE at $\emptyset_{P P / P E} \sim 0.15 \%$ on the Si substrates with different solvent evaporation rates. Crystalline layers, substrate-oriented crystals, randomly oriented crystals, and amorphous assemblies were all observed. Incident angle $\alpha=0.8^{\circ}$ for solvent 
evaporation rate $=1.0 \mu \mathrm{L} / \mathrm{min}$ and $\alpha=0.14^{\circ}$ for other conditions. d, bcc lattice with (110) planes oriented in parallel with the substrate. e, Phase diagram of the assembled morphology as a function of solvent evaporation rate and polyolefin concentration.

\section{Electron Tomography and Reconstruction on a Single PGNP Crystal}

Scanning transmission electron microscopy (STEM) tomography was performed to characterize the 3-D spatial organization of the PGNPs in a single crystal formed on the substrate ${ }^{34}$. The reconstruction method and additional details can be found in the methods section. The reconstructed cluster shows a 3-D disk shape composed of stacked PGNP layers. A slight decrease in the number of PGNPs in each layer from the bottom to the top is observed. Within the reconstructed crystal, the layers closer to the top and the bottom (substrate) planes have more defects and grain boundaries (Fig. 3a). Figure 3b shows the two base plane layers plotted in different colors, demonstrating a locally distorted hexagonal lattice in each plane. The registration between the layers is predominantly bridge sites, and thus is consistent with bcc stacking and the base plane is (110). The in-plane orientation, i.e. the (001) direction, is mostly in the horizontal as demonstrated, but varies close to the crystal edge. As shown in the enlarged area in Fig. $\mathbf{3 b}$, the orientation is rotated by roughly 60 or -60 degrees. Similar registration is also seen in the base planes near to the top, where a hollow site stacking can be observed near the edge (SI Fig. S21). PGNP organization in these regions thus probably reflects the structural transition from hexagonal packing to a bcc lattice during crystal growth. The fact that such organization is more prominent at edges and top layers indicates that the crystal grows from the centre to the periphery. Out-ofplane structure is examined by analysing PGNP stacking in the vertical direction. Fig. $\mathbf{3 c}$ is a cut slice that lies vertically in the crystal with two consecutive PGNP layers depicted in different colors. The figure on the right shows where the slice cut is from the top view. The layers are identified as 
bcc (001) planes to reflect the majority orientation. Their horizontals are the [-110] direction and their verticals are the [110] direction. The image in the middle depicts projection of one of the layers, which clearly shows the square lattice of the bcc (001) plane. Based on the structural characterization, we can confirm that the structure of crystals formed on carbon substrates is bcc, and thus identical to the structure of crystals formed in solution. The crystal orientation is biased by the surface yielding (110) base planes. However, unlike the truncated Wolff polyhedral formed by substrate-bound DNA-grafted NPs ${ }^{12}$, the defects present on the crystal's surface and its rounded shape reveal that the crystal growth is limited by the short-range PGNP diffusion at the late stage of the solvent evaporation. This is unique for the PGNP system with low cohesive energy and is thus advantageous to the future studies on the kinetic pathways of crystallization.

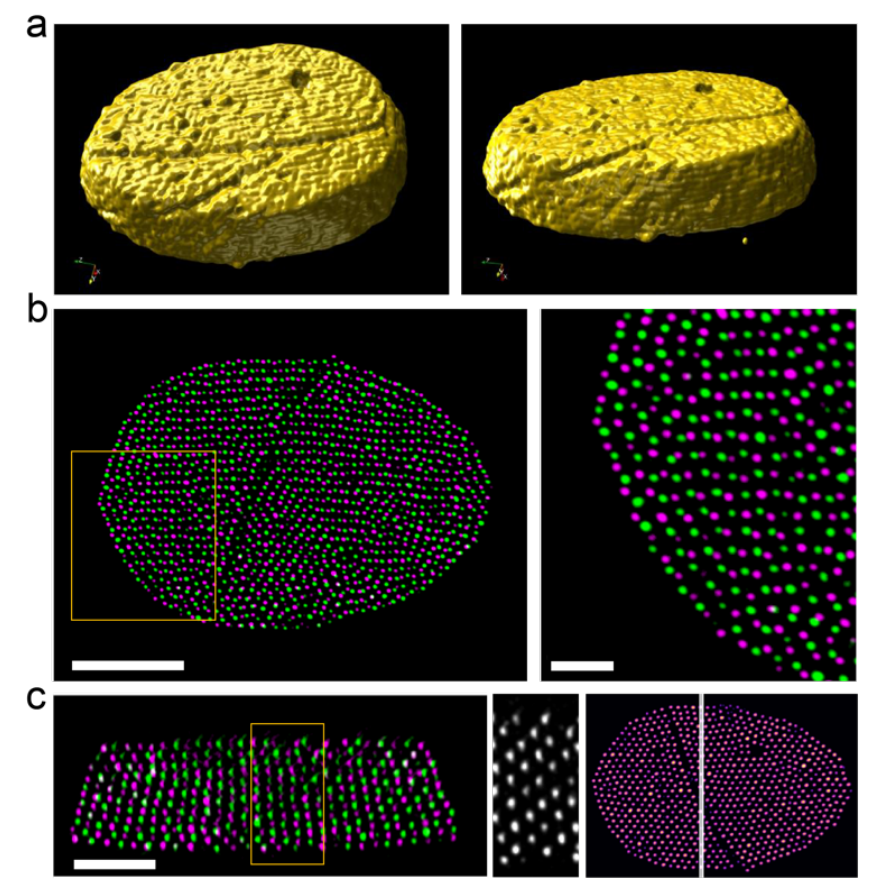

Fig. 3 | Electron tomography and reconstruction of a single bec crystal composed of (5.3k) Au-PS 0. 60 NPs on the substrate. PP/PE was added at $\emptyset_{P P / P E} \sim 0.1 \%$. a, 3-D visualization of the crystal surface (see Methods) clearly illustrating its overall shape and the defective top layers. b, Two base plane layers plotted in green and purple, demonstrating a distorted hexagonal lattice in 
each plane. The registration between the layers is predominantly bridge sites, as seen in the enlarged view on the right. Scale bars, $100 \mathrm{~nm}$ and $25 \mathrm{~nm}$. c, Two consecutive planes within a vertical slice plotted in green and purple. PGNPs in one plane sit at the center of the PGNP squares in the other plane. The square lattice of one of the bcc (001) planes is shown in the enlarged view in the middle. The image on the right shows where the cut lies in the top view. Scale bar, $50 \mathrm{~nm}$.

\section{Pattern PGNP Crystals on Substrates}

PGNP crystal growth is most readily controlled via the solvent evaporation rate, but crystallization kinetics can also be modulated by changing the NP size. Individual small clusters composed of $25 \mathrm{~nm}$ PGNPs were found at $\emptyset_{P P / P E} \sim 0.05 \%$ (Fig. 4a). Decreased cluster size is attributed to the decreased diffusivity of larger particles according to the Stokes-Einstein equation. The formation of dimers, trimers, tetramers, pentamers as well as hexamers were observed (SI Fig. S22). Such geometries of metal nanostructures have received great attention in the past years because of their unique applications in photocatalysis, surface-enhanced spectroscopies, and nonlinear optics ${ }^{35}$. Nevertheless, specific clustering of two to six PGNPs has been a major challenge and was previously realized through elaborate templating strategies ${ }^{36-39}$. It is worthwhile to further explore the possibilities of separating these nanostructures at high purity.

Low-magnification TEM images demonstrate that the growth of 3-D crystals is not a rare event and is highly reproducible. At $\emptyset_{P P / P E} \sim 0.05 \%$, the sizes of the crystals can be fitted to a normal distribution with an average diameter of around $165 \mathrm{~nm}$. The size refers to the diameter of a disk with the area as the crystal: $d=2 \sqrt{\operatorname{area} / \pi}(2)$, where $d$ is the diameter. TEM images were further analyzed by Voronoi tessellation to visualize the corresponding occupied area of each cluster. Fig. 4b shows the 2D map of Voronoi cells which are color-coded based on their area. The nucleation sites were randomly distributed. Fitting the linear model to the Voronoi cell area versus the size of the corresponding crystals gives a slope of (6.8 \pm 1.9$)$ and an intercept of $(6.3 \pm 1.4) \times 10^{4} \mathrm{~nm}^{2}$ (Fig. 
4d). The linear correlation between the two suggests that a $2 \mathrm{D}$ self-assembly model can be applied $^{11}$. Once a nuclei forms, the surrounding area is depleted of PGNPs, and further growth of the crystal relies on the short-range diffusion and local rearrangements of PGNPs.

Furthermore, the capability to control the PGNP nucleation and growth process is promising for producing hierarchical assemblies ${ }^{40-42}$. Patterned surfaces can be used as templates to control the location of NP arrays taking advantage of various interactions between the NPs and substrates. As an example, PGNPs can be self-assembled into a square array of crystals by using templated carbon films (Fig. 4e). The commercially available carbon-coated TEM grids have orthogonally patterned vacuum holes of $\sim 1 \mu \mathrm{m}$ diameter and $\sim 650 \mathrm{~nm}$ separation. The available spaces between the holes are comparable to the size of PGNP crystals formed. Here, the nucleation of PGNPs preferentially took place between the holes, and the crystals were thus organized into a square lattice (Fig. 4g and 4h). Crystallinity was maintained within individual clusters (SI Fig. S23). At least two periodicities existed: the several nanometer inter-PGNP distance in each cluster and the micrometer separation between clusters. Furthermore, the PGNPs preferred two possible nucleation sites: either between two adjacent vacancies or at interstitial sites of the hole lattice (see blue dots in Fig. 4f). These gave two distinctive periodicities and cluster sizes, due to the differences in space available to grow crystals. The average diameter of the crystals formed are $600 \mathrm{~nm}$ and $\sim 900 \mathrm{~nm}$, respectively. 


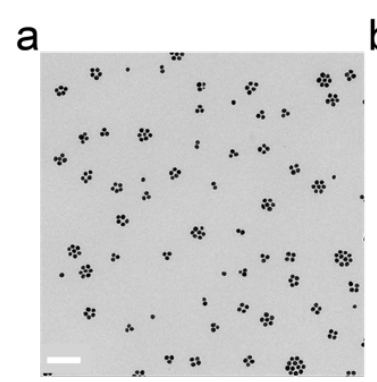

e

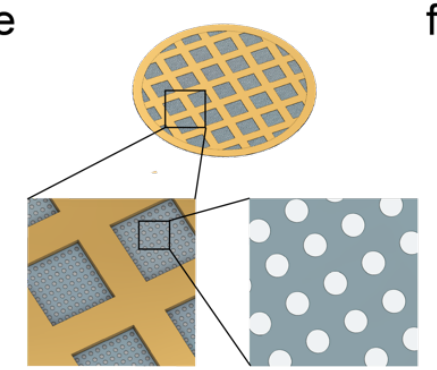

$b$

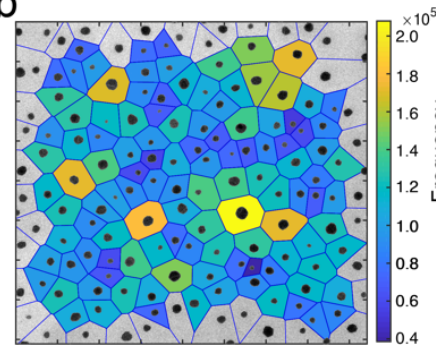

f

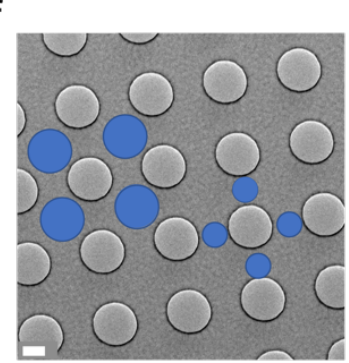

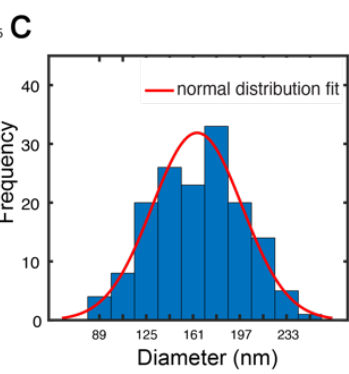
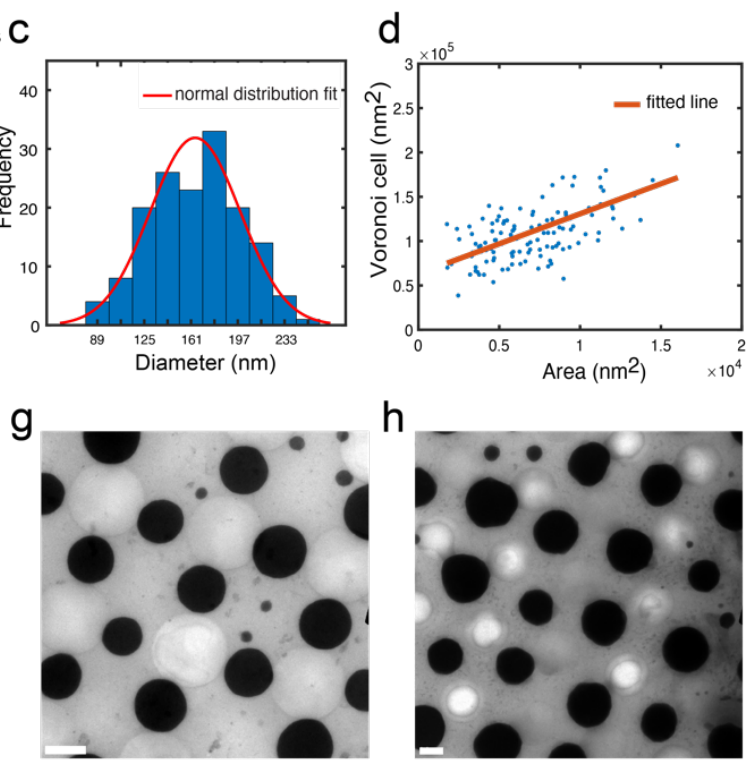

$\mathrm{h}$

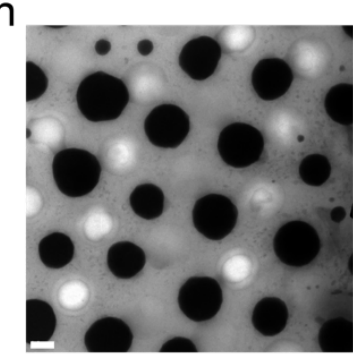

Fig. 4 | Controlled PGNP crystallization on substrates. a, PGNP clusters assembled from $25 \mathrm{~nm} \mathrm{Au} \mathrm{NPs} \mathrm{(PS}=6 \mathrm{k}$ ) with PP/PE at $\emptyset_{P P / P E} \sim 0.05 \%$. Scale bar, $200 \mathrm{~nm}$. b, TEM image of (1.7k) Au-PS $\mathrm{S}_{0.70} \mathrm{NP}$ crystals formed with PP/PE at $\emptyset_{P P / P E} \sim 0.08 \%$ and Voronoi representation color-coded by the Voronoi cell area in 2D. c, Crystal size distribution plot, fitted with a normal distribution curve. d, Voronoi cell area as a function of crystal size in 2D, fitted with a linear curve. $(1.7 \mathrm{k}) \mathrm{Au}-\mathrm{PS}_{0.70} \mathrm{NP}$ crystals characterized in $\mathbf{c}$ and $\mathbf{d}$ were formed with $\mathrm{PP} / \mathrm{PE}_{1}$ at $\emptyset_{P P / P E} \sim 0.08 \%$. e, Schematic illustration of the templated carbon films with orthogonally arrayed holes mounted on TEM grids. Two types of different possible nucleation sites are highlighted with blue circles in f. $\mathbf{g},(1.7 \mathrm{k}) \mathrm{Au}-\mathrm{PS}_{0.70} \mathrm{NP}$ crystals formed at bridge sites between adjacent holes. Scale bar, 500nm. h, (1.7k) Au-PS 0.70 NP crystals formed at center sites of the hole lattice. PP/PE was added at $\emptyset_{P P / P E} \sim 0.15 \%$.

\section{Conclusions}

We demonstrated that a properly chosen polymer precipitate and PGNP surface modification can effectively change NP/solvent interactions, leading to a rather controlled nucleation of PGNPs in dilute solutions. The initial PGNP clustering occurs in solution due to the precipitation of precipitants onto PGNP surfaces. The crystallization process is further modulated by manipulating solvent evaporation rate, NP size and nucleation sites. Such a robust growth of superlattice crystals 
within several minutes opens up possibilities of NP crystallization into complicated confined structures, which should be applicable to other systems as well. These PGNP systems are ideal for future studies in crystallization at single building block level since the NP assembly's energetic driving force, kinetics and pathways can be easily modulated. Last but not least, these PGNP crystals are obtained with the presence of trace amount of impurity from plastic containers that are routinely used. The results showed here clearly confirmed the importance to identify any potential chemical impurity and their effects in the field of nanoscience, even at a trace amount. 


\section{Methods}

Materials. Hydrogen tetrachloroaurate (III) trihydrate $\left(\mathrm{HAuCl}_{4} \bullet 3 \mathrm{H}_{2} \mathrm{O}\right.$, Sigma-Aldrich, $99.995 \%$, trace metals basis), oleylamine (OAm) (Sigma-Aldrich, technical grade, 70\%), tert-butylamineborane (TBAB) complex (Aldrich, 97\%), 1,2,3,4-tetrahydronaphthalene (tetralin, anhydrous, 99\%), styrene (ReagentPlus ${ }^{\circledR}$, contains 4-tert-butylcatechol as stabilizer, $\geq 99 \%$ ), 2-Cyano-2propyl benzodithioate (chain transfer agent) (Sigma-Aldrich, >97\% (HPLC)), 2,2'-Azobis(2methylpropionitrile) (AIBN) (Sigma-Aldrich, 98\%), hydrazine (Sigma-Aldrich, anhydrous, 98\%), methanol (Fisher Scientific, analytical reagent grade), chloroform (Sigma-Aldrich, contains 100200 ppm amylenes as stabilizer, $\geq 99.5 \%$ ) and acetone (Sigma-Aldrich) were used in this work.

Olefin mixtures are dissolved from VWR high performance microcentrifuge tubes, polypropylene, 1.5ml. Carboxylic acid terminated poly(N-isopropylacrylamide) (PNIPAM-COOH, $\mathrm{Mn}=6.3$ $\mathrm{kg} / \mathrm{mol}, \mathrm{PDI}=1.4)$. Carboxylic acid terminated polybutadiene $(\mathrm{PBd}-\mathrm{COOH}, \mathrm{Mn}=2.5 \mathrm{~kg} / \mathrm{mol}$, PDI $=1.3)$. polystyrene-b-poly(cholesteryloxycarbonyloxy ethyl methacrylate) (PS-b-PHEMAC, $\mathrm{Mn}=29 \mathrm{~kg} / \mathrm{mol}$ for PS, $28 \mathrm{~kg} / \mathrm{mol}$ for PHEMAC, PDI = 1.06) were purchased from Polymer Source Inc.

Synthesis of PGNPs. 3.8nm OAm-stabilized Au NPs were synthesized according to literature methods ${ }^{43}$. The synthesis was carried out under nitrogen atmosphere using standard Schlenk line techniques. Thiol-terminated PS (PS-SH) was synthesized using Radical Addition Fragmentation Transfer (RAFT) polymerization and end-functionalized by aminolysis. Au NPs grafted with PSSH were prepared by a ligand exchange process as reported before ${ }^{24}$.

PGNP crystal growth upon solvent evaporation. Au PGNPs were dispersed in toluene. All solution has the $\emptyset_{\mathrm{NP}}=0.1 \%$ before solvent evaporation. Polymer additives were dissolved in toluene and added to the PGNP solution at $\emptyset_{\text {polymer }}$ ranging from $0.01 \%-0.5 \%$ before solvent 
evaporation. After solvent evaporates, samples on silicon wafers and TEM grids were used for GISAXS measurements and TEM analysis, respectively. For crystal growth on normal substrates, a certain amount of solution (50ul) is drop casted on 200 mesh $\mathrm{Cu}$ TEM grids or a Silicon wafer and dried after around 20min. For a quick-drying experiment, less than 5ul solution is drop casted and the solution dries within $1 \mathrm{~min}$. Solvent evaporation was done under ambient condition without specific control. For crystal growth on templated TEM grids, slow solvent evaporation is desired in order to get highly ordered crystals. The TEM grid was placed in a Teflon well with the dimension of $1.5 \mathrm{~cm} * 1.5 \mathrm{~cm} * 1.0 \mathrm{~cm}$ covered with glass slide. Sample dries completely after 2 hours.

Transmission electron microscopy (TEM). Au PGNP samples prepared upon solvent evaporation were imaged using a FEI Tecnai 12 at the accelerating voltages of $120 \mathrm{kV}$.

Small angel X-ray Scattering (SAXS). In-situ solution SAXS experiments were performed at Beamline 11-BM (Complex Material Scattering) of National Synchrotron Light Source-II (NSLSII, Brookhaven National Laboratory). The SAXS data were collected on a Dectris 2M detector at a sample-to-detector distance of $2 \mathrm{~m}$, using an X-ray beam with an energy of $13.5 \mathrm{keV}$ (the corresponding wavelength $\lambda=0.92 \AA$ ). Au PGNP solution was loaded in a tube and was positioned so that the beam went through the solution. The solution was left dry under ambient environment and X-ray was exposed every $30 \mathrm{~s}$. The q range is $0.001 \sim 0.2^{\circ}$.

Static SAXS experiments were performed at beamline 7.3.3 at Advanced Light Source (ALS) in Lawrence Berkeley National Laboratory. The X-ray wavelength is $1.24 \AA$. The scattered X-ray intensity distribution was detected using a high-speed detector, Pilatus 2M. Images were plotted as intensities (I) vs q, where $\mathrm{q}=(4 \pi / \lambda) \sin (\theta), \lambda$ is the wavelength of the incident $\mathrm{X}$-ray beam, and $2 \theta$ is the scattering angle. 
Peak Deconvolution. The scattering peaks were deconvoluted using a Matlab command-line peak fitting program(43). The interested area containing the sharp diffraction peak at $\mathrm{q} \sim 0.1 \AA^{-1}$ from PGNP crystals and the broad peak from PGNP clusters in the solution was selected to fit a twopeak Gaussian model. At least 10 trial fits were performed and the best one with lowest fitting error was selected. Data points were collected as the integration ratio of the two deconvoluted peaks.

Grazing incidence/transmission small angle x-ray scattering (GI(T)SAXS). GI(T)SAXS experiments at an incident angle $\alpha$ of $0.14^{\circ} \sim 0.8^{\circ}$ were conducted at beam line 8-ID-E at the Advanced Photon Source (APS) of Argonne National Laboratory. Samples were drop-casted on a $1 \mathrm{~cm} * 1 \mathrm{~cm}$ silicon substrate with $100-500 \mathrm{ul}$ solvent reservoir placed in the house-made solvent annealing chamber. The chamber was sealed and N2 flow was purged through to control the solvent evaporation. The scattered X-ray intensity distribution was detected using a high-speed detector, Pilatus 1M. Samples were exposed to $7.35-\mathrm{keV}$ radiation $(\lambda=1.68 \AA)$.

Scanning transmission electron microscopy (STEM) tomography. The tomography experiment was performed using a FEI Titan microscope operated at $300 \mathrm{kV}$. The HAADF-STEM images were acquired with a $10 \mathrm{mrad}$ probe semi- convergence angle. The projections from the 3.0nm (3.8k) Au NPs was accomplished over the tilt range of $-70^{\circ}$ to $70^{\circ}$, with $2^{\circ}$ step size and pixel size of $0.63 \mathrm{~nm}$. The alignment was performed on IMOD (version 4.9.10) making use of fiducial markers and custom python scripts. Reconstructions were performed using inverse radon transformation from the skimage python library.

Acknowledgements: This work was funded by the U.S. Department of Energy, Office of Science, Office of Basic Energy Sciences, Materials Sciences and Engineering Division under Contract No. 
DE-AC02-05- CH11231 (Organic-Inorganic Nanocomposites KC3104). Nanoparticle and polymer characterizations done at the Molecular Foundry were supported by the Office of Science, Office of Basic Energy Sciences, of the U.S. Department of Energy under Contract No. DE-AC0205CH11231. Scattering studies were done at the Advanced Light Source is supported by the U.S. Department of Energy, Office of Science, Office of Basic Energy Sciences, of the U.S. Department of Energy under Contract DE-AC02-05CH11231 and at Advanced Photon Source, use of the Advanced Photon Source was supported by the US Department of Energy, Office of Science, Office of Basic Energy Sciences, under Contract DE-AC02-06CH1135. Author contributions: TX conceived the idea and guided the project. YQ performed studies contributed to Figure 1, 2, and 4. EY performed nanoparticle synthesis. ADS performed STEM tomography in Figure 3. CLA performed GIWAXS and HT-GPC measurements. ADS, WT and PE performed tomography analysis. 


\section{References:}

1. Manoharan, V.N., Colloidal matter: Packing, geometry, and entropy. Science, 2015. 349(6251): p. 1253751.

2. Ou, Z., Z. Wang, B. Luo, E. Luijten, and Q. Chen, Kinetic pathways of crystallization at the nanoscale. Nature materials, 2019: p. 1-6.

3. Grzelczak, M., J. Vermant, E.M. Furst, and L.M. Liz-Marzán, Directed self-assembly of nanoparticles. ACS nano, 2010. 4(7): p. 3591-3605.

4. Boles, M.A., M. Engel, and D.V. Talapin, Self-assembly of colloidal nanocrystals: from intricate structures to functional materials. Chemical reviews, 2016. 116(18): p. 1122011289.

5. Alivisatos, A.P., et al., Organization of'nanocrystal molecules' using DNA. Nature, 1996. 382(6592): p. 609-611.

6. Mirkin, C.A., R.L. Letsinger, R.C. Mucic, and J.J. Storhoff, A DNA-based method for rationally assembling nanoparticles into macroscopic materials. Nature, 1996. 382(6592): p. 607-609.

7. Nykypanchuk, D., M.M. Maye, D. Van Der Lelie, and O. Gang, DNA-guided crystallization of colloidal nanoparticles. Nature, 2008. 451(7178): p. 549-552.

8. Mullin, J.W., Crystallization. 2001: Elsevier.

9. Hoover, W.G. and F.H. Ree, Melting transition and communal entropy for hard spheres. The Journal of Chemical Physics, 1968. 49(8): p. 3609-3617.

10. Dong, A., J. Chen, P.M. Vora, J.M. Kikkawa, and C.B. Murray, Binary nanocrystal superlattice membranes self-assembled at the liquid-air interface. Nature, 2010. 466(7305): p. 474.

11. Bigioni, T.P., et al., Kinetically driven self assembly of highly ordered nanoparticle monolayers. Nature materials, 2006. 5(4): p. 265.

12. Lewis, D.J., L.Z. Zornberg, D.J. Carter, and R.J. Macfarlane, Single-crystal Winterbottom constructions of nanoparticle superlattices. Nature Materials, 2020: p. 1-6.

13. Park, J.e., et al., Surfactant-Assisted Emulsion Self-Assembly of Nanoparticles into Hollow Vesicle-Like Structures and 2D Plates. Advanced Functional Materials, 2016. 26(43): p. 7791-7798.

14. Zhuang, J., H. Wu, Y. Yang, and Y.C. Cao, Supercrystalline colloidal particles from artificial atoms. Journal of the American Chemical Society, 2007. 129(46): p. 1416614167.

15. Jones, A.G. and J. Mullin, Programmed cooling crystallization of potassium sulphate solutions. Chemical Engineering Science, 1974. 29(1): p. 105-118.

16. Sun, J.-K., Y.I. Sobolev, W. Zhang, Q. Zhuang, and B.A. Grzybowski, Enhancing crystal growth using polyelectrolyte solutions and shear flow. Nature, 2020. 579(7797): p. 73-79.

17. Kulkarni, A. and C. Zukoski, Depletion interactions and protein crystallization. Journal of crystal growth, 2001. 232(1-4): p. 156-164.

18. McPherson, A., [5] Crystallization of macromolecules: General principles, in Methods in enzymology. 1985, Elsevier. p. 112-120.

19. Song, R.-Q. and H. Cölfen, Additive controlled crystallization. CrystEngComm, 2011. 13(5): p. 1249-1276.

20. Sauter, A., et al., On the question of two-step nucleation in protein crystallization. Faraday discussions, 2015. 179: p. 41-58. 
21. Dumetz, A.C., A.M. Snellinger-O'Brien, E.W. Kaler, and A.M. Lenhoff, Patterns of protein-protein interactions in salt solutions and implications for protein crystallization. Protein Science, 2007. 16(9): p. 1867-1877.

22. Akcora, P., et al., Anisotropic self-assembly of spherical polymer-grafted nanoparticles. Nature materials, 2009. 8(4): p. 354-359.

23. Kumar, S.K., N. Jouault, B. Benicewicz, and T. Neely, Nanocomposites with polymer grafted nanoparticles. Macromolecules, 2013. 46(9): p. 3199-3214.

24. Ye, X., et al., Structural diversity in binary superlattices self-assembled from polymergrafted nanocrystals. Nature Communications, 2015. 6: p. 10052.

25. Fernandes, N.J., H. Koerner, E.P. Giannelis, and R.A. Vaia, Hairy nanoparticle assemblies as one-component functional polymer nanocomposites: opportunities and challenges. Mrs Communications, 2013. 3(1): p. 13-29.

26. Choi, J., et al., Effect of Polymer-Graft Modification on the Order Formation in Particle Assembly Structures. Langmuir, 2013. 29(21): p. 6452-6459.

27. Choi, J., H. Dong, K. Matyjaszewski, and M.R. Bockstaller, Flexible Particle Array Structures by Controlling Polymer Graft Architecture. Journal of the American Chemical Society, 2010. 132(36): p. 12537-12539.

28. Nie, Z., et al., Self-assembly of metal-polymer analogues of amphiphilic triblock copolymers. Nature materials, 2007. 6(8): p. 609-614.

29. Choueiri, R.M., et al., Surface patterning of nanoparticles with polymer patches. Nature, 2016. 538(7623): p. 79-83.

30. Josten, E., et al., Superlattice growth and rearrangement during evaporation-induced nanoparticle self-assembly. Scientific reports, 2017. 7(1): p. 1-9.

31. Mahadevapuram, N., J. Strzalka, and G.E. Stein, Grazing-incidence transmission small angle $X$-ray scattering from thin films of block copolymers. Journal of Polymer Science Part B: Polymer Physics, 2013. 51(7): p. 602-610.

32. Deegan, R.D., et al., Capillary flow as the cause of ring stains from dried liquid drops. Nature, 1997. 389(6653): p. 827-829.

33. Marechal, M., M. Hermes, and M. Dijkstra, Stacking in sediments of colloidal hard spheres. The Journal of chemical physics, 2011. 135(3): p. 034510.

34. Ercius, P., O. Alaidi, M.J. Rames, and G. Ren, Electron tomography: a three-dimensional analytic tool for hard and soft materials research. Advanced materials, 2015. 27(38): p. 5638-5663.

35. Chumanov, G., K. Sokolov, B.W. Gregory, and T.M. Cotton, Colloidal metal films as a substrate for surface-enhanced spectroscopy. The Journal of Physical Chemistry, 1995. 99(23): p. 9466-9471.

36. Loweth, C.J., W.B. Caldwell, X. Peng, A.P. Alivisatos, and P.G. Schultz, DNA-based assembly of gold nanocrystals. Angewandte Chemie International Edition, 1999. 38(12): p. 1808-1812.

37. Novak, J.P. and D.L. Feldheim, Assembly of phenylacetylene-bridged silver and gold nanoparticle arrays. Journal of the American Chemical Society, 2000. 122(16): p. 39793980.

38. Yin, Y., Y. Lu, B. Gates, and Y. Xia, Template-assisted self-assembly: a practical route to complex aggregates of monodispersed colloids with well-defined sizes, shapes, and structures. Journal of the American Chemical Society, 2001. 123(36): p. 8718-8729. 
39. Wang, X., et al., Polymer-encapsulated gold-nanoparticle dimers: facile preparation and catalytical application in guided growth of dimeric ZnO-nanowires. Nano letters, 2008. 8(9): p. 2643-2647.

40. Zhong, Z., B. Gates, Y. Xia, and D. Qin, Soft lithographic approach to the fabrication of highly ordered $2 D$ arrays of magnetic nanoparticles on the surfaces of silicon substrates. Langmuir, 2000. 16(26): p. 10369-10375.

41. Whitesides, G.M. and B. Grzybowski, Self-assembly at all scales. Science, 2002. 295(5564): p. 2418-2421.

42. Cui, Y., et al., Integration of colloidal nanocrystals into lithographically patterned devices. Nano Letters, 2004. 4(6): p. 1093-1098.

43. Peng, S., et al., A facile synthesis of monodisperse Au nanoparticles and their catalysis of CO oxidation. Nano research, 2008. 1(3): p. 229-234.

44. Tom O'Haver (2020). peakfit.m (https://www.mathworks.com/matlabcentral/fileexchange /23611-peakfit-m), MATLAB Central File Exchange. 